TRANSACTIONS OF THE

AMERICAN MATHEMATICAL SOCIETY

Volume 352, Number 1, Pages 115-132

S 0002-9947(99)02497-6

Article electronically published on July 1, 1999

\title{
THE SPECTRUM OF INFINITE REGULAR LINE GRAPHS
}

\author{
TOMOYUKI SHIRAI
}

\begin{abstract}
Let $G$ be an infinite $d$-regular graph and $L(G)$ its line graph. We consider discrete Laplacians on $G$ and $L(G)$, and show the exact relation between the spectrum of $-\Delta_{G}$ and that of $-\Delta_{L(G)}$. Our method is also applicable to $\left(d_{1}, d_{2}\right)$-semiregular graphs, subdivision graphs and para-line graphs.
\end{abstract}

\section{INTRODUCTION}

Many authors have intensively studied the spectra of the Laplacians (or adjacency matrices) of finite graphs and the relationship to the structure and characteristic properties of graphs (cf. [1]). Recently, the spectra of Laplacians of infinite graphs have been studied in the various frameworks, for example, harmonic analysis on graphs, probability theory, especially Markov chains, and potential theory, and so on. A survey of the topic can be found in [5]. In [3] the transience of the Markov chains on a graph and its line graph has been studied, and also an inequality for the bottoms of the spectrum of the discrete Laplacian on a regular graph and its line graph has been given.

In this paper we give, in place of the inequality, the exact relation between the spectra of the Laplacians on regular graphs and their line graphs. Also we will show similar relations for some other graphs, such as semiregular graphs, subdivision graphs and para-line graphs.

In order to state our theorems, we prepare some definitions and notations. A graph $G$ is a pair $(V(G), E(G))$ of a set $V(G)$ and a set $E(G)$ of unordered pairs $x y$ of two distinct points $x, y$ of $V(G)$. The sets $V(G)$ and $E(G)$ are called the vertex set and the edge set of $G$, respectively. We define a neighborhood set of a vertex $x$ by

$$
N_{x}=\{y \in V(G) ; x y \in E(G)\} .
$$

The degree of a vertex $x$ is the cardinality of $N_{x}$ and is denoted by $m(x)$. Throughout this paper, we assume that an infinite graph $G$ is simple, connected and locally finite, that is, $G$ has no self-loops and no multiple edges, $G$ has a path from $x$ to $y$ for any two distinct vertices $x, y \in V(G)$ and $m(x)<\infty$ for any $x \in V(G)$.

Received by the editors July 12, 1998 .

1991 Mathematics Subject Classification. Primary 39A12; Secondary 39A70.

Key words and phrases. Regular line graph, subdivision, para-line graph, discrete Laplacian, spectrum.

(C)1999 American Mathematical Society 
We denote by $\ell^{2}(G)$ an $\ell^{2}$-space of functions on $V(G)$ with the inner product defined by

$$
\langle f, g\rangle_{G}=\sum_{x \in V(G)} m(x) f(x) g(x) .
$$

Now we define a discrete Laplacian which acts on $\ell^{2}(G)$ as follows:

$$
\Delta_{G} f(x)=\frac{1}{m(x)} \sum_{y \in N_{x}} f(y)-f(x),
$$

where $N_{x}$ is the neighborhood of a vertex $x$. We denote the spectrum of $-\Delta_{G}$ by $\operatorname{Spec}\left(-\Delta_{G}\right)$.

Remark 1.1. In this definition one can easily check that $\Delta_{G}$ is a bounded selfadjoint operator with $\operatorname{Spec}\left(-\Delta_{G}\right) \subset[0,2]$, where both 0 and 2 cannot be eigenvalues because $G$ is infinite.

A graph $G$ is called $d$-regular if $m(x)=d$ for all $x \in V(G)$. The line graph $L(G)$ of a graph $G$ is defined as follows:

- $V(L(G))=E(G)$,

- $E(L(G))=\{(x y)(y z) ; x y \in E(G)$ and $y z \in E(G), x \neq z\}$.

(See Figure 2.1.)

Our first theorem is the following:

Theorem 1.2. Let $d \geq 3$. Let $G$ be an infinite $d$-regular graph and $L(G)$ the line graph of $G$. Then,

$$
\operatorname{Spec}\left(-\Delta_{L(G)}\right)=\frac{d}{2 d-2} \operatorname{Spec}\left(-\Delta_{G}\right) \cup\left\{\frac{d}{d-1}\right\}
$$

where $\frac{d}{d-1}$ is an eigenvalue with infinite multiplicity.

We can define the $n$-th line graph of a graph $G$ inductively by

$$
\begin{aligned}
L^{0}(G) & =G, \\
L^{n}(G) & =L\left(L^{n-1}(G)\right) \text { for } n \geq 1 .
\end{aligned}
$$

Note that the line graph of a regular graph is also regular, and so the $n$-th line graph $L^{n}(G)$ is regular for each $n \geq 0$.

Example 1.3. Let $G$ be the 2-dimensional square lattice $\mathbf{Z}^{2}$. It is easy to see that $\operatorname{Spec}\left(\mathbf{Z}^{2}\right)=\operatorname{Spec}\left(-\Delta_{\mathbf{Z}^{2}}\right)=[0,2]$ by Fourier series. Then applying Theorem 1.2 to this case repeatedly, we have

$$
\begin{aligned}
\operatorname{Spec}\left(\mathbf{Z}^{2}\right) & =[0,2], \\
\operatorname{Spec}\left(L\left(\mathbf{Z}^{2}\right)\right) & =[0,4 / 3] \cup\{4 / 3\}, \\
\operatorname{Spec}\left(L^{2}\left(\mathbf{Z}^{2}\right)\right) & =[0,4 / 5] \cup\{4 / 5\} \cup\{6 / 5\}, \\
\operatorname{Spec}\left(L^{3}\left(\mathbf{Z}^{2}\right)\right) & =[0,4 / 9] \cup\{4 / 9\} \cup\{2 / 3\} \cup\{10 / 9\}, \\
\cdots &
\end{aligned}
$$

Here all the eigenvalues are of infinite multiplicity. 
Example 1.4. Let $T_{d}$ be a $d$-regular tree $(d \geq 3)$. It is well-known that $\operatorname{Spec}\left(T_{d}\right)=$ $\operatorname{Spec}\left(-\Delta_{T_{d}}\right)=\left[\lambda_{0}, \lambda_{\infty}\right]$ where $\lambda_{0}=1-\frac{2 \sqrt{d-1}}{d}$ and $\lambda_{\infty}=1+\frac{2 \sqrt{d-1}}{d}$. Then applying Theorem 1.2 to this case repeatedly, we have

$$
\begin{aligned}
\operatorname{Spec}\left(T_{d}\right) & =\left[\lambda_{0}, \lambda_{\infty}\right], \\
\operatorname{Spec}\left(L\left(T_{d}\right)\right) & =\frac{d}{2 d-2}\left[\lambda_{0}, \lambda_{\infty}\right] \cup\left\{\frac{d}{d-1}\right\}, \\
\operatorname{Spec}\left(L^{2}\left(T_{d}\right)\right) & =\frac{d}{4 d-6}\left[\lambda_{0}, \lambda_{\infty}\right] \cup\left\{\frac{d}{2 d-3}\right\} \cup\left\{\frac{2 d-2}{2 d-3}\right\}, \\
\operatorname{Spec}\left(L^{3}\left(T_{d}\right)\right) & =\frac{d}{8 d-14}\left[\lambda_{0}, \lambda_{\infty}\right] \cup\left\{\frac{d}{4 d-7}\right\} \cup\left\{\frac{2 d-2}{4 d-7}\right\} \cup\left\{\frac{4 d-6}{4 d-7}\right\}, \\
\ldots &
\end{aligned}
$$

We note that the maximal eigenvalues $\frac{d}{d-1}, \frac{2 d-2}{2 d-3}, \ldots$ converge to 1 .

For a semiregular graph (the definition will be given in Section 3), we can show a similar relation:

Theorem 1.5. Let $G$ be an infinite $\left(d_{1}, d_{2}\right)$-semiregular graph where $d_{1} \geq d_{2} \geq 3$ or $d_{1}>d_{2}=2$, and $f_{ \pm}^{\left(d_{1}, d_{2}\right)}(x)=\left(d_{1}+d_{2} \pm \sqrt{\left(d_{1}-d_{2}\right)^{2}+4 d_{1} d_{2}(1-x)^{2}}\right) / 2 D$, where $D=d_{1}+d_{2}-2$. Let $\operatorname{Spec}^{*}\left(-\Delta_{G}\right)=\overline{\operatorname{Spec}\left(-\Delta_{G}\right) \backslash\{1\}}$. Then

$$
\operatorname{Spec}\left(-\Delta_{L(G)}\right)=f_{-}^{\left(d_{1}, d_{2}\right)}\left(\operatorname{Spec}^{*}\left(-\Delta_{G}\right)\right) \cup S \cup f_{+}^{\left(d_{1}, d_{2}\right)}\left(\operatorname{Spec}^{*}\left(-\Delta_{G}\right)\right) \cup\left\{\frac{d_{1}+d_{2}}{D}\right\}
$$

where $\frac{d_{1}+d_{2}}{D}$ is an eigenvalue with infinite multiplicity, and $S \subset\left\{f_{ \pm}^{\left(d_{1}, d_{2}\right)}(1)\right\}=$ $\left\{\frac{d_{1}}{D}, \frac{d_{2}}{D}\right\}$ which is determined by eigenfunctions of $-\Delta_{G}$ corresponding to $\{1\}$.

More precise description of the set $S$ will be given in Theorem 3.2.

The next theorem shows that line graphs have special spectral property (see [4]).

Theorem 1.6. Let $G$ be an infinite graph such that $\sup _{x \in V(G)} m(x)=M<\infty$. Let $\lambda_{\infty}^{e s s}$ be the upper bound of the essential spectrum of $-\Delta_{G}$. Then

$$
1+\frac{2}{M} \leq \lambda_{\infty}^{e s s}
$$

and the equality holds if $G$ is a $M$-regular line graph.

A similar technique as in the proofs of Theorem 1.2 and Theorem 1.5 can be also applied to other kinds of graph. The first one is the subdivision $S(G)$ of a graph $G$, whose definition will be found in Section 4 .

Theorem 1.7. Let $d \geq 3$. Let $G$ be an infinite d-regular graph, and $f_{ \pm}^{S}(x)=$ $1 \pm \sqrt{1-x / 2}$. Then

$$
\operatorname{Spec}\left(-\Delta_{S(G)}\right)=f_{-}^{S}\left(\operatorname{Spec}\left(-\Delta_{G}\right)\right) \cup\{1\} \cup f_{+}^{S}\left(\operatorname{Spec}\left(-\Delta_{G}\right)\right)
$$

where 1 is an eigenvalue with infinite multiplicity.

The second one is the para-line graph $p-L(G)$ of a graph $G$ which was introduced in [3] in order to show a relationship between the behavior of simple random walks on a graph and its line graph. The definition of para-line graph will be given in Section 5. Since a para-line graph can be regarded as a line graph of the subdivision 
of $G$ and the subdivision of a $d$-regular graph is $(d, 2)$-semiregular, from Theorem 1.5 and Theorem 1.7, we have

$$
\begin{aligned}
& \operatorname{Spec}\left(-\Delta_{p-L(G)}\right) \\
& =f_{-}^{(d, 2)}\left(\overline{\operatorname{Spec}\left(-\Delta_{S(G)}\right) \backslash\{1\}}\right) \cup S \cup f_{+}^{(d, 2)}\left(\overline{\operatorname{Spec}\left(-\Delta_{S(G)}\right) \backslash\{1\}}\right) \cup\left\{\frac{d+2}{d}\right\} \\
& =f_{-}^{(d, 2)}\left(f_{ \pm}^{S}\left(\operatorname{Spec}\left(-\Delta_{G}\right)\right)\right) \cup S \cup f_{+}^{(d, 2)}\left(f_{ \pm}^{S}\left(\operatorname{Spec}\left(-\Delta_{G}\right)\right)\right) \cup\left\{\frac{d+2}{d}\right\} \\
& =f_{-}^{p}\left(\operatorname{Spec}\left(-\Delta_{G}\right)\right) \cup S \cup f_{+}^{p}\left(\operatorname{Spec}\left(-\Delta_{G}\right)\right) \cup\left\{\frac{d+2}{d}\right\},
\end{aligned}
$$

where $S \subset f_{ \pm}^{(d, 2)}(1)=\left\{1, \frac{2}{d}\right\}$ and $f_{ \pm}^{p}=f_{ \pm}^{(d, 2)} \circ f_{ \pm}^{S}$ is the function defined in Theorem 1.8 .

The set $S$ is not characterized precisely on this stage. However, owing to the structure of para-line graphs, we have the following theorem:

Theorem 1.8. Let $d \geq 3$. Let $G$ be an infinite d-regular graph and $p-L(G)$ its para-line graph, and $f_{ \pm}^{p}(x)=\left(d+2 \pm \sqrt{(d+2)^{2}-4 d x}\right) / 2 d$. Then

$$
\operatorname{Spec}\left(-\Delta_{p-L(G)}\right)=f_{-}^{p}\left(\operatorname{Spec}\left(-\Delta_{G}\right)\right) \cup\{1\} \cup f_{+}^{p}\left(\operatorname{Spec}\left(-\Delta_{G}\right)\right) \cup\left\{\frac{d+2}{d}\right\}
$$

where 1 and $\frac{d+2}{d}$ are eigenvalues with infinite multiplicity.

Thus, in this case $S=\left\{\frac{d_{1}}{D}\right\}=\{1\}$ in Theorem 1.5. In general, all four cases that $S=\emptyset,\left\{\frac{d_{1}}{D}\right\},\left\{\frac{d_{2}}{D}\right\}$, or $\left\{\frac{d_{1}}{D}, \frac{d_{2}}{D}\right\}$ may occur.

Our theorems are also applicable to finite graphs [1]. The spectrum of the Laplacian on a (finite) pre-Sierpinski gasket can be obtained from Theorem 1.2 and Theorem 1.8.

Remark 1.9. Let $K^{4}$ be a complete graph on 4 vertices and $F_{n}$ a pre- $n$-Sierpinski gasket (see Figure 1.1). It is easy to check that

$$
L\left((p-L)^{n}\left(K^{4}\right)\right)=F_{n+1} \cup F_{n} / \sim .
$$

Here by $\sim$, we identify three vertices 1,2 and 3 of $F_{n+1}$ with those of $F_{n}$ respectively. Then we obtain a similar result as studied in [7]. It follows from the form of the functions $f_{ \pm}^{p}$ that the spectrum of pre- $n$-Sierpinski gasket has Cantor structure as $n \rightarrow \infty$.
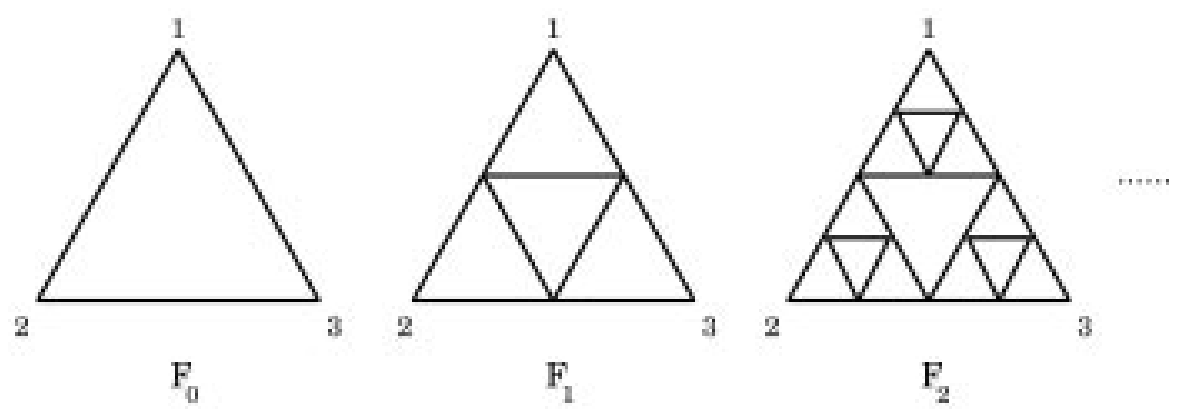

Figure 1.1. Pre-Sierpinski gasket. 
We will repeatedly use the following lemma due to Weyl (see [6]) in the proofs of the theorems above.

Lemma 1.10. (Weyl's criterion) Let $H$ be a separable Hilbert space, and let $L$ be a bounded self-adjoint operator on $H$. Then $\lambda \in \operatorname{Spec}(L)$ if and only if there exists a sequence $\left\{f_{n}\right\}_{n=1}^{\infty}$ so that $\left\|f_{n}\right\|=1$ and $\lim _{n \rightarrow \infty}\left\|(L-\lambda) f_{n}\right\|=0$.

\section{LINE GRAPHS OF REGULAR GRAPHS}

The line graph $L(G)$ of a graph $G$ is the graph such that its vertex set is $E(G)$ and two vertices are adjacent if and only if they have exactly one common vertex of $G$ (see Figure 2.1).

Remark 2.1. The line graph of a $d$-regular graph is $(2 d-2)$-regular.

Then we obtain the following:

Theorem 2.2. Let $d \geq 3$. Let $G$ be an infinite $d$-regular graph and $L(G)$ the line graph of $G$. Then,

$$
\operatorname{Spec}\left(-\Delta_{L(G)}\right)=\frac{d}{2 d-2} \operatorname{Spec}\left(-\Delta_{G}\right) \cup\left\{\frac{d}{d-1}\right\}
$$

where $\frac{d}{d-1}$ is an eigenvalue with infinite multiplicity.



G

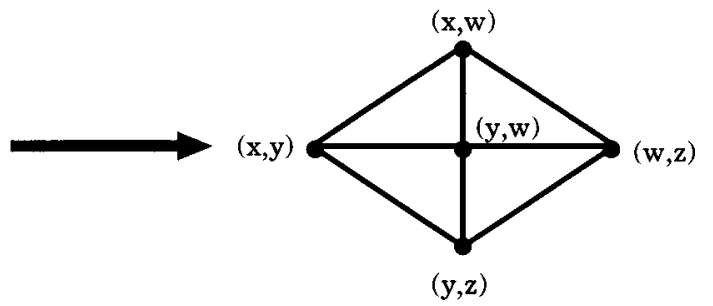

$\mathrm{L}(\mathrm{G})$

Figure 2.1. Line graph.

This theorem depends much on the algebraic relation between two Laplacians (Lemma 2.4).

We identify $L(G)$ with $\{(x, y) \in V(G) \times V(G) ; x y \in E(G)\} / \sim$, where $(x, y) \sim$ $(y, x)$. So, we can regard $\ell^{2}(L(G))$ as symmetric square integrable functions on the set above. Then, we can write down $\Delta_{L(G)}$ using this notation as follows:

$$
\begin{aligned}
\Delta_{L(G)} F(x, y) & =\frac{1}{2 d-2} \sum_{(r, s) \in N_{(x, y)}} F(r, s)-F(x, y) \\
& =\frac{1}{2 d-2}\left(\sum_{r \in N_{x}}(F(x, r)-F(x, y))+\sum_{r \in N_{y}}(F(r, y)-F(x, y))\right) .
\end{aligned}
$$


Now, we define two operators, $\phi: \ell^{2}(G) \rightarrow \ell^{2}(L(G))$ and $\phi^{*}: \ell^{2}(L(G)) \rightarrow \ell^{2}(G)$ in the following way:

$$
\begin{aligned}
\phi f(x, y) & =\sqrt{\frac{d}{2 d-2}}(f(x)+f(y)), \\
\phi^{*} F(x) & =\sqrt{\frac{2 d-2}{d}} \sum_{r \in N_{x}} F(x, r) .
\end{aligned}
$$

Lemma 2.3. The operator $\phi^{*}$ is the adjoint operator of $\phi$, that is,

$$
\langle F, \phi f\rangle_{L(G)}=\left\langle\phi^{*} F, f\right\rangle_{G} .
$$

In particular, for any $f, g \in \ell^{2}(G)$ and any $F, G \in \ell^{2}(L(G))$,

$$
\begin{aligned}
\langle\phi f, \phi g\rangle_{L(G)} & =\left\langle\phi^{*} \phi f, g\right\rangle_{G}, \\
\left\langle\phi^{*} F, \phi^{*} G\right\rangle_{G} & =\left\langle\phi \phi^{*} F, G\right\rangle_{L(G)} .
\end{aligned}
$$

Proof. First we note that

$$
\sum_{x y \in E(G)} F(x, y)=\frac{1}{2} \sum_{x \in G} \sum_{y \in N_{x}} F(x, y)=\frac{1}{2} \sum_{y \in G} \sum_{x \in N_{y}} F(x, y) .
$$

By (2.1) we have

$$
\begin{aligned}
\langle F, \phi f\rangle_{L(G)} & =\sum_{x y \in E(G)}(2 d-2) F(x, y) \sqrt{\frac{d}{2 d-2}}(f(x)+f(y)) \\
& =\sqrt{d(2 d-2)} \sum_{x \in V(G)} \sum_{r \in N_{x}} F(x, r) f(x) \\
& =\sum_{x \in V(G)} d f(x) \phi^{*} F(x) \\
& =\left\langle\phi^{*} F, f\right\rangle_{G} .
\end{aligned}
$$

The following lemma is essential for the theorem.

Lemma 2.4. Two operators $\phi$ and $\phi^{*}$ are linear bounded operators, and have the following relations:

$$
\begin{aligned}
& \text { 1) } \phi^{*} \phi=d\left(\Delta_{G}+2\right), \\
& \text { 2) } \phi \phi^{*}=(2 d-2)\left(\Delta_{L(G)}+\left(\frac{d}{d-1}\right)\right), \\
& \text { 3) } \Delta_{L(G)} \phi=\frac{d}{2 d-2} \phi \Delta_{G} \\
& \text { 4) } \phi^{*} \Delta_{L(G)}=\frac{d}{2 d-2} \Delta_{G} \phi^{*} .
\end{aligned}
$$

Proof. 1) By (2.1) we obtain

$$
\begin{aligned}
\left(\phi^{*} \phi f\right)(x) & =\sqrt{\frac{2 d-2}{d}} \sum_{r \in N_{x}} \phi(f)(x, r)=\sum_{r \in N_{x}}(f(x)+f(r)) \\
& =\sum_{r \in N_{x}}((f(r)-f(x))+2 f(x))=d\left(\Delta_{G}+2\right) f(x) .
\end{aligned}
$$


2) Also by (2.1)

$$
\begin{aligned}
\left(\phi \phi^{*} F\right)(x, y) & \\
= & \sqrt{\frac{d}{2 d-2}}\left(\phi^{*} F(x)+\phi^{*} F(y)\right)=\sum_{r \in N_{x}} F(x, r)+\sum_{r \in N_{y}} F(y, r) \\
= & \sum_{r \in N_{x}}(F(x, r)-F(x, y))+\sum_{r \in N_{y}}(F(y, r)-F(x, y))+2 d F(x, y) \\
= & (2 d-2) \Delta_{L(G)} F(x, y)+2 d F(x, y) \\
= & (2 d-2)\left(\Delta_{L(G)}+\frac{d}{d-1}\right) F(x, y) .
\end{aligned}
$$

3) and 4) are obvious from 1) and 2).

Proposition 2.5. Let $H_{1}$ and $H_{2}$ be separable Hilbert spaces, and let $L_{1}$ and $L_{2}$ be bounded self-adjoint operators on $H_{1}$ and $H_{2}$, respectively. Suppose $L_{1}$ is a positive operator, 0 is not an eigenvalue of $L_{1}$ and there exists a bounded operator $\phi$ from $\mathrm{H}_{1}$ to $\mathrm{H}_{2}$ which satisfies the following two conditions:

$$
\begin{aligned}
& \text { 1) } \phi L_{1}=L_{2} \phi, \\
& \text { 2) } \inf _{\|f\|=1}\|\phi f\| \geq C>0 \text { or } L_{1}=P\left(\phi^{*} \phi\right),
\end{aligned}
$$

where $\phi^{*}$ is the adjoint operator of $\phi$ and $P$ is a continuous function such that $0 \leq P(x) \leq K x$ on $\operatorname{Spec}\left(L_{1}\right)$ for some $K>0$. Then,

$$
\operatorname{Spec}\left(L_{1}\right)=\operatorname{Spec}\left(\left.L_{2}\right|_{\overline{\phi\left(H_{1}\right)}}\right) .
$$

Proof. Assume that $\lambda \in \operatorname{Spec}\left(L_{1}\right)$ and $\lambda \neq 0$. Then there exists a sequence $\left\{f_{n}\right\}_{n \geq 1}$ such that $\left\|f_{n}\right\|=1$ and $\left\|\left(\lambda-L_{1}\right) f_{n}\right\| \rightarrow 0(n \rightarrow \infty)$ by Lemma 1.10. (From now on we will often use Lemma 1.10 in this way.) Since $\left\|\phi\left(\lambda-L_{1}\right) f_{n}\right\|=\left\|\left(\lambda-L_{2}\right) \phi f_{n}\right\| \rightarrow 0$ as $n \rightarrow \infty$, in order to show $\lambda \in \operatorname{Spec}\left(L_{2}\right)$, by Lemma 1.10, we check that $\left\|\phi f_{n}\right\|$ is bounded from below for sufficient large $n$. When $\inf _{\|f\|=1}\|\phi f\| \geq C>0$, it is trivial. Thus we consider the case when $L_{1}=P\left(\phi^{*} \phi\right)$. By the assumption on $P$,

$$
\begin{aligned}
\left\langle\left(\lambda-L_{1}\right) f,\left(\lambda-L_{1}\right) f\right\rangle & \geq \lambda^{2}\|f\|^{2}-2 \lambda\left\langle L_{1} f, f\right\rangle \\
& =\lambda^{2}\|f\|^{2}-2 \lambda\left\langle P\left(\phi^{*} \phi\right) f, f\right\rangle \\
& \geq \lambda^{2}\|f\|^{2}-2 \lambda K\left\langle\phi^{*} \phi f, f\right\rangle,
\end{aligned}
$$

and so we have

$$
\|\phi f\|^{2} \geq \frac{\lambda}{2 K}\|f\|^{2}-\frac{1}{2 K \lambda}\left\|\left(\lambda-L_{1}\right) f\right\|^{2} .
$$

Therefore for sufficiently large $n$,

$$
\begin{aligned}
\left\|\phi f_{n}\right\|^{2} & \geq \frac{\lambda}{2 K}\left\|f_{n}\right\|^{2}-\frac{1}{2 K \lambda}\left\|\left(\lambda-L_{1}\right) f_{n}\right\|^{2} \\
& \geq \frac{\lambda}{4 K},
\end{aligned}
$$

since $\left\|\left(\lambda-L_{1}\right) f_{n}\right\| \rightarrow 0$. So $\operatorname{Spec}\left(L_{1}\right) \backslash\{0\} \subset \operatorname{Spec}\left(\left.L_{2}\right|_{\overline{\phi\left(H_{1}\right)}}\right)$. However, since spectrum sets are closed and 0 is not an eigenvalue of $L_{1}, \overline{\operatorname{Spec}\left(L_{1}\right) \backslash\{0\}}=\operatorname{Spec}\left(L_{1}\right) \subset$ $\operatorname{Spec}\left(\left.L_{2}\right|_{\overline{\phi\left(H_{1}\right)}}\right)$. 
Conversely, we will show

$$
\operatorname{Spec}\left(L_{2} \mid \overline{\phi\left(H_{1}\right)}\right) \subset \operatorname{Spec}\left(L_{1}\right) .
$$

Assume that $\lambda \in \operatorname{Spec}\left(\left.L_{2}\right|_{\overline{\phi\left(H_{1}\right)}}\right)$. If $\inf _{\|f\|=1}\|\phi f\| \geq C>0$, since $\left\|\left(L_{2}-\lambda\right) \phi f_{n}\right\|=$ $\left\|\phi\left(L_{1}-\lambda\right) f_{n}\right\| \rightarrow 0$ as $n \rightarrow \infty,\left\|\left(L_{1}-\lambda\right) f_{n}\right\| \rightarrow 0$. Then $\lambda \in \operatorname{Spec}\left(L_{1}\right)$. Let us consider the case that $L_{1}=P\left(\phi^{*} \phi\right)$. Put $H_{1, \delta}=E([\delta, \infty)) H_{1}$ where $E([a, b])$ is the resolution of the identity for the operator $L_{1}$. For any $f \in H_{1, \delta}$ we obtain

$$
\delta\|f\|^{2} \leq\left\langle L_{1} f, f\right\rangle=\left\langle P\left(\phi^{*} \phi\right) f, f\right\rangle \leq K\|\phi f\|^{2},
$$

and

$$
\|\phi f\| \geq \sqrt{\frac{\delta}{K}}\|f\| .
$$

Since $\left(L_{1}-\lambda\right) f \in H_{1, \delta}$ for $f \in H_{1, \delta}$, by Lemma 1.10, we have

$$
\operatorname{Spec}\left(\left.L_{2}\right|_{\phi\left(H_{1, \delta}\right)}\right) \subset \operatorname{Spec}\left(\left.L_{1}\right|_{H_{1, \delta}}\right) \subset \operatorname{Spec}\left(L_{1}\right) .
$$

As $\delta>0$ is arbitrary and 0 is not an eigenvalue, we have

$$
\operatorname{Spec}\left(\left.L_{2}\right|_{\phi\left(H_{1}\right)}\right)=\overline{\bigcup_{\delta>0} \operatorname{Spec}\left(\left.L_{2}\right|_{\phi\left(H_{1, \delta}\right)}\right)} \subset \operatorname{Spec}\left(L_{1}\right) .
$$

This is the desired conclusion.

Now, we decompose $\ell^{2}(L(G))$ into two closed subspaces $E$ and $E^{\perp}$ where $E=$ $\overline{\phi\left(\ell^{2}(G)\right)}$. Note that $\Delta_{L(G)}$ preserves $E$ and $E^{\perp}$ by Lemma 2.42$)$ and 3). First, we consider the spectrum of $-\left.\Delta_{L(G)}\right|_{E}$.

Proposition 2.6. Let $E$ be as above and $d \geq 2$. Then

$$
\operatorname{Spec}\left(-\left.\Delta_{L(G)}\right|_{E}\right)=\frac{d}{2 d-2} \operatorname{Spec}\left(-\Delta_{G}\right) .
$$

Proof. Set $L_{1}=d\left(\Delta_{G}+2\right), L_{2}=(2 d-2)\left(\Delta_{L(G)}+\frac{d}{d-1}\right), H_{1}=\ell^{2}(G)$, and $H_{2}=$ $\ell^{2}(L(G))$. The operator $\phi$ is the one defined by (2.1). It is obvious that $\phi, L_{1}$ and $L_{2}$ satisfy the conditions in Proposition 2.5 by Lemma 2.3 and Lemma 2.4, and that $L_{1}$ is a positive operator and does not have 0 as an eigenvalue by Remark 1.1. So, we obtain

$$
\operatorname{Spec}\left(\left.(2 d-2)\left(\Delta_{L(G)}+\frac{d}{d-1}\right)\right|_{E}\right)=\operatorname{Spec}\left(d\left(\Delta_{G}+2\right)\right),
$$

and this implies the proposition.

Next, we characterize the space $E^{\perp}$.

Proposition 2.7. Let $d \geq 2$. Then

$$
E^{\perp}=\operatorname{ker} \phi^{*}=\left\{F \in \ell^{2}(L(G)) ;-\Delta_{L(G)} F=\frac{d}{d-1} F\right\} .
$$

If $d=2, E^{\perp}$ is empty. If $d \geq 3, E^{\perp}$ is infinite dimensional, that is, $\Delta_{L(G)}$ restricted to $E^{\perp}$ has an eigenvalue with infinite multiplicity and in particular,

$$
\operatorname{Spec}\left(-\left.\Delta_{L(G)}\right|_{E^{\perp}}\right)=\left\{\frac{d}{d-1}\right\} .
$$


Proof. In general, it is easy to see that $F \in E^{\perp}$ is equivalent to $\phi^{*} F=0$, since

$$
F \in E^{\perp} \Leftrightarrow 0=\langle F, \phi f\rangle=\left\langle\phi^{*} F, f\right\rangle \text { for any } f \in \ell^{2}(G) .
$$

Moreover, if $F \in \operatorname{ker} \phi^{*}$, we obtain

$$
0=\phi \phi^{*} F=(2 d-2)\left(\Delta_{L(G)}+\frac{d}{d-1}\right) F
$$

by Lemma 2.4. Suppose $F$ is an eigenfunction of $-\Delta_{L(G)}$ corresponding to $\frac{d}{d-1}$. Then for any $f \in \ell^{2}(G)$

$$
\begin{aligned}
0 & =\left\langle(2 d-2)\left(\Delta_{L(G)}+\frac{d}{d-1}\right) F, \phi f\right\rangle=\left\langle\phi \phi^{*} F, \phi f\right\rangle \\
& =\left\langle\phi^{*} F, \phi^{*} \phi f\right\rangle=\left\langle\phi^{*} F, d\left(\Delta_{G}+2\right) f\right\rangle .
\end{aligned}
$$

Since 2 is not an eigenvalue of $-\Delta_{G}$, so $\left(\Delta_{G}+2\right) \ell^{2}(G)$ is dense in $\ell^{2}(G)$. Therefore, $\phi^{*} F=0$, that is, $F \in \operatorname{ker} \phi^{*}$.

When $d=2$, by Remark 1.1, it is trivial that $E^{\perp}$ is empty. Thus, we assume that $d \geq 3$. Before proving that $E^{\perp}$ is an infinite dimensional eigenspace corresponding to $\frac{d}{d-1}$, we prepare a lemma.

Lemma 2.8. Let $\delta_{x y} \in \ell^{2}(L(G))$ be the indicator function of a vertex $x y \in V(L(G))$ and $\delta_{x}, \delta_{y} \in \ell^{2}(G)$ the indicator functions of vertices $x, y$, respectively. Then,

$$
\left(\Delta_{L(G)}+\frac{d}{d-1}\right) \delta_{x y}=\sqrt{\frac{1}{d(2 d-2)}} \phi\left(\delta_{x}+\delta_{y}\right) .
$$

Proof. Observe that

$$
\phi^{*} \delta_{x y}=\sqrt{\frac{2 d-2}{d}}\left(\delta_{x}+\delta_{y}\right)
$$

Applying $\phi$ to both sides of the equation above and using Lemma 2.4 2), we obtain the lemma.

Corollary 2.9. Let $\gamma=x_{0} x_{1} \cdots x_{2 n-1}$ be an even closed path in $G$ and set

$$
F_{\gamma}=\sum_{k=0}^{2 n-1}(-1)^{k} \delta_{x_{k} x_{k+1}}
$$

where $x_{2 n}=x_{0}$. Then, $F_{\gamma} \in \operatorname{ker} \phi^{*}$. In particular, if $F_{\gamma} \neq 0$, then $F_{\gamma}$ is an eigenfunction of $-\Delta_{L(G)}$ corresponding to $\frac{d}{d-1}$.

Proof. Since $x_{0} x_{1} \cdots x_{2 n-1}$ is an even closed path,

$$
\begin{aligned}
\phi^{*} F_{\gamma} & =\phi^{*} \sum_{k=0}^{2 n-1}(-1)^{k} \delta_{x_{k} x_{k+1}} \\
& =\sqrt{\frac{2 d-2}{d}} \sum_{k=0}^{2 n-1}(-1)^{k}\left(\delta_{x_{k}}+\delta_{x_{k+1}}\right) \\
& =0 .
\end{aligned}
$$


Now we will call $\gamma$ a closed walk if $F_{\gamma} \in \operatorname{ker} \phi^{*} \backslash\{0\}$.

We proceed to the second part of the proof of Proposition 2.7. It is divided into three cases according to the structure of a graph $G$.

First, let $G$ be the $d$-regular tree. We can construct eigenfunctions corresponding to $\frac{d}{d-1}$ as follows. Take any vertex $0 \in V(G)$ and fix it. Each vertex $x$ of $G$ can be identified with the shortest path from 0 to $x$ and the set $V(G)$ with

$$
\begin{aligned}
\left\{x=\left(x_{0} x_{1} \ldots x_{n}\right)(n \geq 0) ; x_{0}\right. & =0, x_{1} \in\{1,2, \ldots, d\}, \\
x_{i} & \in\{1,2, \ldots, d-1\}(2 \leq i \leq n)\} .
\end{aligned}
$$

Note that the vertices adjacent to $x=\left(x_{0} x_{1} x_{2} \ldots x_{n}\right)$ for $n \geq 1$ are of the form $\left(x_{0} x_{1} x_{2} \ldots x_{n-1}\right)$ or $\left(x_{0} x_{1} x_{2} \ldots x_{n} a\right)$ with $a \in\{1,2, \ldots, d-1\}$. We define a function $F_{0}$ on $L(G)$ inductively by

$$
\begin{gathered}
F_{0}\left(x_{0}, x_{0} x_{1}\right)=0 \quad \text { for } \quad x_{1} \in\{1,2, \ldots, d\}, \\
F_{0}\left(x_{0} x_{1}, x_{0} x_{1} x_{2}\right)=\left\{\begin{aligned}
1 & \text { if } x_{1}=1, x_{2}=1, \\
-1 & \text { if } x_{1}=1, x_{2}=2, \\
0 & \text { otherwise }
\end{aligned}\right. \\
F_{0}\left(x_{0} x_{1} x_{2} \ldots x_{n}, x_{0} x_{1} x_{2} \ldots x_{n+1}\right)=\frac{-1}{d-1} F_{0}\left(x_{0} x_{1} x_{2} \ldots x_{n-1}, x_{0} x_{1} x_{2} \ldots x_{n}\right) \\
\text { for } n \geq 2 .
\end{gathered}
$$

Obviously, $\phi^{*} F_{0}=0$, that is, $-\Delta_{L(G)} F_{0}=\frac{d}{d-1} F_{0}$. It remains to show that $F_{0} \in$ $\ell^{2}(L(G))$. Indeed,

$$
\begin{aligned}
\left\|F_{0}\right\|_{L(G)}^{2}= & \sum_{x y \in E(G)}(2 d-2) F_{0}(x, y)^{2} \\
= & (2 d-2) \sum_{x_{2}=1}^{2}\left\{F_{0}\left(01,01 x_{2}\right)^{2}\right. \\
& \left.\quad+\sum_{n \geq 3}\left(\sum_{x_{3}, \ldots, x_{n}=1}^{d-1} F_{0}\left(01 x_{2} x_{3} \ldots x_{n-1}, 01 x_{2} x_{3} \ldots x_{n}\right)^{2}\right)\right\} \\
= & 2(2 d-2)\left\{1+\sum_{n \geq 3}(d-1)^{n-2} \cdot\left(\frac{-1}{d-1}\right)^{2(n-2)}\right\} \\
< & \infty .
\end{aligned}
$$

Similarly, by considering the vertex $(x_{0} \overbrace{11 \ldots 1}^{n \text { times }})$ in place of $x_{0}$, we can define $F_{n}$ for $n \geq 1$. By the definition of $\left\{F_{n}\right\}_{n \geq 0}$, they are linearly independent and eigenfunctions corresponding to $\frac{d}{d-1}$. Therefore $E^{\perp}$ is infinite dimensional.

Second, we consider a graph $G$ which has only a finite number of even closed walks. In this case, subtracting a sufficiently large subgraph from $G$ which contains all even closed walks, we obtain a finite number of half infinite trees. Then we can construct eigenfunctions in the same way as above.

Finally, in the other cases, $G$ has an infinite number of even closed walks and then, by Corollary 2.9, we obtain eigenfunctions associated with each even closed walks. 
In any case, $E^{\perp}$ is infinite dimensional. Thus the proof is completed.

Theorem 2.2 follows from Proposition 2.6 and Proposition 2.7.

\section{LINE GRAPHS OF SEMIREGULAR GRAPHS}

A graph $G$ is called a bipartite graph if $G$ has no cycles of odd length; the vertex set $V(G)$ can be partitioned into two sets $V_{1}$ and $V_{2}$ in such a way that every edge in $E(G)$ connects a vertex in $V_{1}$ with a vertex in $V_{2}$. A bipartite graph $G$ with a bipartition $\left\{V_{i}\right\}_{i=1,2}$ is called a $\left(d_{1}, d_{2}\right)$-semiregular graph if the degree of each vertex in $V_{i}$ is the constant $d_{i}(i=1,2)$. Note that a $d$-regular graph is $(d, d)$-semiregular if and only if it is a bipartite graph.

Remark 3.1. The line graph of a $\left(d_{1}, d_{2}\right)$-semiregular graph is $\left(d_{1}+d_{2}-2\right)$-regular.

The Laplacian on $L(G)$ is given by

$\Delta_{L(G)} F(x, y)=\frac{1}{d_{1}+d_{2}-2}\left(\sum_{r \in N_{x}}(F(x, r)-F(x, y))+\sum_{r \in N_{y}}(F(r, y)-F(x, y))\right)$.

Theorem 3.2. Let $G$ be an infinite $\left(d_{1}, d_{2}\right)$-semiregular graph where $d_{1} \geq d_{2} \geq 3$ or $d_{1}>d_{2}=2$, and $f_{ \pm}^{\left(d_{1}, d_{2}\right)}(x)=\left(d_{1}+d_{2} \pm \sqrt{\left(d_{1}-d_{2}\right)^{2}+4 d_{1} d_{2}(1-x)^{2}}\right) / 2 D$, where $D=d_{1}+d_{2}-2$. Let $\operatorname{Spec}^{*}\left(-\Delta_{G}\right)=\overline{\operatorname{Spec}\left(-\Delta_{G}\right) \backslash\{1\}}$. Then

$$
\operatorname{Spec}\left(-\Delta_{L(G)}\right)=f_{-}^{\left(d_{1}, d_{2}\right)}\left(\operatorname{Spec}^{*}\left(-\Delta_{G}\right)\right) \cup S \cup f_{+}^{\left(d_{1}, d_{2}\right)}\left(\operatorname{Spec}^{*}\left(-\Delta_{G}\right)\right) \cup\left\{\frac{d_{1}+d_{2}}{D}\right\}
$$

where $\frac{d_{1}+d_{2}}{D}$ is an eigenvalue with infinite multiplicity, and $S \subset\left\{f_{ \pm}^{\left(d_{1}, d_{2}\right)}(1)\right\}=$ $\left\{\frac{d_{1}}{D}, \frac{d_{2}}{D}\right\}$. Furthermore, when $d_{1} \neq d_{2}, S$ contains an eigenvalue $\frac{d_{1}}{D}$ (resp. $\frac{d_{2}}{D}$ ) if and only if there exists an eigenvalue 1 of $-\Delta_{G}$ and its corresponding eigenfunction is supported on the set $V_{2}$ (resp. $\left.V_{1}\right)$. Here $V_{i}$ is the set of vertices whose degrees are $d_{i}$. When $d_{1}=d_{2}=d, S=\left\{\frac{d}{D}\right\}$ if there exists an eigenvalue 1 of $-\Delta_{G}$.

In order to prove this theorem we define two operators $\phi: \ell^{2}(G) \rightarrow \ell^{2}(L(G))$ and $\phi^{*}: \ell^{2}(L(G)) \rightarrow \ell^{2}(G)$ by

$$
\begin{aligned}
\phi f(x, y) & =f(x)+f(y), \\
\phi^{*} F(x) & =\frac{D}{m(x)} \sum_{r \in N_{x}} F(x, r) .
\end{aligned}
$$

Lemma 3.3. The operator $\phi^{*}$ is the adjoint operator of $\phi$, i.e.,

$$
\langle F, \phi f\rangle_{L(G)}=\left\langle\phi^{*} F, f\right\rangle_{G}
$$

Proof. By (3.1) we have

$$
\begin{aligned}
\langle F, \phi f\rangle_{L(G)} & =\sum_{x \in V(G)} \sum_{r \in N_{x}} D F(x, r) f(x) \\
& =\sum_{x \in V(G)} m(x)\left(\frac{D}{m(x)} \sum_{r \in N_{x}} F(x, r)\right) f(x) \\
& =\left\langle\phi^{*} F, f\right\rangle_{G} .
\end{aligned}
$$


Lemma 3.4. Two operators $\phi$ and $\phi^{*}$ are linear bounded operators, and have the following relations:

1) $\phi^{*} \phi=D\left(\Delta_{G}+2\right)$,

2) $\phi\left(m \phi^{*}\right)=D\left(D \Delta_{L(G)}+(D+2)\right)$, in particular, $\phi\left(m\left(\Delta_{G}+2\right)\right)=\left(D \Delta_{L(G)}+(D+2)\right) \phi$,

3) $\quad\left(\Delta_{G}+1\right) m=\tilde{m}\left(\Delta_{G}+1\right)$,

4) $D \Delta_{L(G)} \phi=\phi \Delta_{G} \tilde{m}$,

5) $\quad D\left(D \Delta_{L(G)}{ }^{2}+(D+2) \Delta_{L(G)}\right) \phi=\phi d_{1} d_{2}\left(\Delta_{G}\left(\Delta_{G}+2\right)\right)$,

where $D=d_{1}+d_{2}-2$ and $\tilde{m}=D+2-m$.

Proof. 1) By (3.1) we have

$$
\begin{aligned}
\phi^{*} \phi f(x) & =\frac{D}{m(x)} \sum_{r \in N_{x}} \phi f(x, r) \\
& =\frac{D}{m(x)} \sum_{r \in N_{x}}(f(r)-f(x)+2 f(x)) \\
& =D\left(\Delta_{G}+2\right) f(x) .
\end{aligned}
$$

2) For any $F \in \ell^{2}(L(G))$

$$
\begin{aligned}
\phi\left(m \phi^{*} F\right)(x, y)= & D\left\{\sum_{r \in N_{x}} F(x, r)+\sum_{r \in N_{y}} F(r, y)\right\} \\
= & D\left\{\sum_{r \in N_{x}}(F(x, r)-F(x, y))+\sum_{r \in N_{y}}(F(r, y)-F(x, y))\right. \\
& \left.+\left(d_{1}+d_{2}\right) F(x, y)\right\} \\
= & D\left\{D \Delta_{L(G)} F(x, y)+(D+2) F(x, y)\right\} .
\end{aligned}
$$

In particular, when $F=\phi f$, using 1), we have

$$
\phi\left(m\left(\Delta_{G}+2\right) f\right)=\left(D \Delta_{L(G)}+(D+2)\right) \phi f .
$$

3) Since $m(r)=\tilde{m}(x)$ for any $r \in N_{x}$, it is trivial.

4) Using 1), 2) and 3), we have

$$
\begin{aligned}
D \Delta_{L(G)} \phi & =\frac{1}{D} \phi m \phi^{*} \phi-(D+2) \phi \\
& =\phi m\left(\Delta_{G}+2\right)-\phi(D+2) \\
& =\phi\left(\left(\Delta_{G}+1\right) \tilde{m}+m\right)-\phi(m+\tilde{m}) \\
& =\phi \Delta_{G} \tilde{m} .
\end{aligned}
$$

5) Using 1), 2) and 4) we obtain

$$
\begin{aligned}
D & \left(D \Delta_{L(G)}^{2}+(D+2) \Delta_{L(G)}\right) \phi \\
& =\Delta_{L(G)} \phi m \phi^{*} \phi \\
& =\frac{1}{D} \phi \Delta_{G} \tilde{m} m D\left(\Delta_{G}+2\right) \\
& =\phi d_{1} d_{2} \Delta_{G}\left(\Delta_{G}+2\right) .
\end{aligned}
$$


Lemma 3.5. Let $G$ be a bipartite graph. Then $\operatorname{Spec}\left(-\Delta_{G}\right)$ is symmetric with respect to 1 , that is, $1+\mu \in \operatorname{Spec}\left(-\Delta_{G}\right)$ is equivalent to $1-\mu \in \operatorname{Spec}\left(-\Delta_{G}\right)$ for any $0 \leq \mu \leq 1$.

Proof. Suppose $1+\mu \in \operatorname{Spec}\left(-\Delta_{G}\right)$; we can take a sequence $\left\{f_{n}\right\}_{n>1}$ such that $\left\|f_{n}\right\|=1$ and $\left\|\left(-\Delta_{G}-(1+\mu)\right) f_{n}\right\| \rightarrow 0$ as $n \rightarrow \infty$. Since $G$ is bipartite, for each $f_{n}$, we can define another function by

$$
\tilde{f}_{n}=\left\{\begin{aligned}
f_{n} & \text { on } V_{1}, \\
-f_{n} & \text { on } V_{2},
\end{aligned}\right.
$$

where $V(G)=V_{1} \cup V_{2}$. It is easy to see that $\left\|\left(-\Delta_{G}-(1-\mu)\right) \tilde{f}_{n}\right\| \rightarrow 0$ as $n \rightarrow \infty$. Then $1-\mu \in \operatorname{Spec}\left(-\Delta_{G}\right)$.

Before proving Theorem 3.2, in the same manner as in Section 2, we decompose $\ell^{2}(L(G))$ into the direct sum of three closed subspaces. Let $W_{1}=\{f \in$ $\left.\ell^{2}(G) ;-\Delta_{G} f=f\right\}, W_{0}=W_{1}^{\perp}$, and $E_{1}=\phi\left(W_{1}\right)$. We put

$$
\ell^{2}(L(G))=E_{0} \oplus E_{1} \oplus E_{2},
$$

where $E_{0}=\overline{\phi\left(\ell^{2}(G)\right)} \ominus E_{1}$, and $E_{2}=\left(E_{0} \oplus E_{1}\right)^{\perp}$ is the orthogonal complement of $\overline{\phi\left(\ell^{2}(G)\right)}$ in $\ell^{2}(L(G))$. It is easy to check by using Lemma 3.4 that $E_{0}=\overline{\phi\left(W_{0}\right)}$ and $\Delta_{L(G)}$ leaves $E_{i}$ 's invariant for $i=0,1,2$.

Proof of Theorem 3.2. First we consider the spectrum of $-\left.\Delta_{L(G)}\right|_{E_{0}}$. Put

$$
\lambda_{ \pm}=\lambda_{ \pm}(\mu)=\frac{d_{1}+d_{2} \pm \sqrt{\left(d_{1}-d_{2}\right)^{2}+4 d_{1} d_{2} \mu^{2}}}{2 D} .
$$

For $0<\mu \leq 1$, let $1+\mu \in \operatorname{Spec}\left(-\Delta_{G}\right)$. (Note that also $1-\mu \in \operatorname{Spec}\left(-\Delta_{G}\right)$ by Lemma 3.5.) Take a sequence $\left\{f_{n}\right\}_{n \geq 1}$ such that $\left\|\left(\Delta_{G}+(1+\mu)\right) f_{n}\right\| \rightarrow 0$ and $\left\|f_{n}\right\|=1$. By Lemma 3.45$)$,

$$
D^{2}\left(\Delta_{L(G)}+\lambda_{+}\right)\left(\Delta_{L(G)}+\lambda_{-}\right) \phi=d_{1} d_{2} \phi\left(\Delta_{G}+(1+\mu)\right)\left(\Delta_{G}+(1-\mu)\right) .
$$

It follows from (3.3) that $\left(\Delta_{L(G)}+\lambda_{+}\right)\left(\Delta_{L(G)}+\lambda_{-}\right) \phi f_{n} \rightarrow 0(n \rightarrow \infty)$. Then if $\left(\Delta_{L(G)}+\lambda_{\mp}\right) \phi f_{n}$ is bounded from below, $\lambda_{ \pm} \in \operatorname{Spec}\left(-\Delta_{L(G)}\right)$. Observe that by Lemma 3.42$)$,

$$
\left\langle\left(\Delta_{L(G)}+\lambda_{-}\right) \phi f_{n}, \phi f_{n}\right\rangle=\left\langle m\left(\Delta_{G}+2\right) f_{n},\left(\Delta_{G}+2\right) f_{n}\right\rangle-\lambda_{+}\left\|\phi f_{n}\right\|^{2} .
$$

Since $\left(\Delta_{G}+(1+\mu)\right) f_{n} \rightarrow 0(n \rightarrow \infty)$, we have

$$
\begin{aligned}
&\left\|D\left(\Delta_{L(G)}+\lambda_{-}\right) \phi f_{n}\right\|^{2} \\
&= D^{2}\left\langle\left(\Delta_{L(G)}+\lambda_{+}\right)\left(\Delta_{L(G)}+\lambda_{-}\right) \phi f_{n}, \phi f_{n}\right\rangle \\
&-D^{2}\left(\lambda_{+}-\lambda_{-}\right)\left\langle\left(\Delta_{L(G)}+\lambda_{-}\right) \phi f_{n}, \phi f_{n}\right\rangle \\
&= d_{1} d_{2}\left\langle\phi\left(\Delta_{G}+(1-\mu)\right)\left(\Delta_{G}+(1+\mu)\right) f_{n}, \phi f_{n}\right\rangle \\
&+D^{2}\left(\lambda_{+}-\lambda_{-}\right)\left\{\lambda_{+}\left\|\phi f_{n}\right\|^{2}-\left\langle m\left(\Delta_{G}+2\right) f_{n},\left(\Delta_{G}+2\right) f_{n}\right\rangle\right\} \\
&= o(1)+D^{2}\left(\lambda_{+}-\lambda_{-}\right)\left\{D \lambda_{+}(1-\mu)\left\|f_{n}\right\|^{2}-(1-\mu)^{2}\left\langle m f_{n}, f_{n}\right\rangle\right\},
\end{aligned}
$$

where $o(1)$ is the function which tends to 0 as $n \rightarrow \infty$. In the same way, we obtain

$$
\begin{aligned}
& \left\|D\left(\Delta_{L(G)}+\lambda_{+}\right) \phi \tilde{f}_{n}\right\|^{2} \\
& \quad=o(1)+D^{2}\left(\lambda_{+}-\lambda_{-}\right)\left\{(1+\mu)^{2}\left\langle m f_{n}, f_{n}\right\rangle-D \lambda_{-}(1+\mu)\left\|f_{n}\right\|^{2}\right\},
\end{aligned}
$$


since $\left(\Delta_{G}+(1-\mu)\right) \tilde{f}_{n} \rightarrow 0(n \rightarrow \infty)$ and $\left|\tilde{f}_{n}(x)\right|=\left|f_{n}(x)\right|$ for any $x \in V(G)$, where $\tilde{f}_{n}$ is defined as in Lemma 3.5. Put

$$
g_{n, \pm}=g_{n, \pm}(\mu)= \pm\left(D \lambda_{ \pm}\left\|f_{n}\right\|^{2}-(1 \mp \mu)\left\langle m f_{n}, f_{n}\right\rangle\right)
$$

and $f_{n}=f_{n, 1}+f_{n, 2}$ where

$$
f_{n, 1}=\left\{\begin{array}{cl}
f_{n} & \text { on } V_{1}, \\
0 & \text { on } V_{2},
\end{array} \quad f_{n, 2}=\left\{\begin{array}{cc}
0 & \text { on } V_{1} \\
f_{n} & \text { on } V_{2}
\end{array}\right.\right.
$$

Then it is easy to see that

$$
g_{n, \pm}= \pm\left[\left(D \lambda_{ \pm}-(1 \mp \mu) d_{1}\right)\left\|f_{n, 1}\right\|^{2}+\left(D \lambda_{ \pm}-(1 \mp \mu) d_{2}\right)\left\|f_{n, 2}\right\|^{2}\right]
$$

and $g_{n, \pm} \geq 0$ for any $0 \leq \mu \leq 1$. Moreover, we can check that if $0<\mu \leq 1$, there exists $\delta=\delta(\mu)>0$ such that

$$
g_{n, \pm} \geq \delta\left\|f_{n}\right\|^{2} .
$$

This implies that $\liminf _{n \rightarrow \infty}\left\|D\left(\Delta_{L(G)}+\lambda_{ \pm}\right) \phi f_{n}\right\| \geq \delta>0$. By Lemma 1.10, we get $\lambda_{ \pm} \in \operatorname{Spec}\left(-\Delta_{L(G)} \mid E_{0}\right)$ and hence $f_{ \pm}^{\left(d_{1}, d_{2}\right)}\left(\operatorname{Spec}^{*}\left(-\Delta_{G}\right)\right) \subset \operatorname{Spec}\left(-\left.\Delta_{L(G)}\right|_{E_{0}}\right)$.

Put $H_{1}=W_{0}, H_{2}=E_{0}, L_{1}=h_{1}\left(-\Delta_{G}\right)$ and $L_{2}=h_{2}\left(-\Delta_{L(G)}\right)$, where $h_{1}(x)=$ $d_{1} d_{2} x(2-x)$ and $h_{2}(x)=D x((D+2)-D x)$. It is easy to see that the assumptions in Proposition 2.5 hold. Then by Proposition 2.5 and spectral mapping theorem we obtain

$$
h_{1}\left(\operatorname{Spec}\left(-\left.\Delta_{G}\right|_{W_{0}}\right)\right)=h_{2}\left(\operatorname{Spec}\left(-\left.\Delta_{L(G)}\right|_{E_{0}}\right)\right)
$$

and so $\operatorname{Spec}\left(-\left.\Delta_{L(G)}\right|_{E_{0}}\right) \subset h_{2}^{-1} \circ h_{1}\left(\operatorname{Spec}^{*}\left(-\Delta_{G}\right)\right)=f_{ \pm}^{\left(d_{1}, d_{2}\right)}\left(\operatorname{Spec}^{*}\left(-\Delta_{G}\right)\right)$. Consequently, we conclude that

$$
\operatorname{Spec}\left(-\left.\Delta_{L(G)}\right|_{E_{0}}\right)=f_{ \pm}^{\left(d_{1}, d_{2}\right)}\left(\operatorname{Spec}^{*}\left(-\Delta_{G}\right)\right) .
$$

Next let us consider the spectrum of $-\left.\Delta_{L(G)}\right|_{E_{1}}$. Let $f \in W_{1}$. Then $\left(\Delta_{G}+1\right) f=$ $0(\mu=0)$. When $d_{1} \neq d_{2}, \lambda_{+}=\frac{d_{1}}{D}, \lambda_{-}=\frac{d_{2}}{D}$. Because of (3.4), (3.5) and (3.6), we obtain

$$
\left\|\left(\Delta_{L(G)}+\lambda_{-}\right) \phi f\right\|=\left.0 \Leftrightarrow f\right|_{V_{2}}=0 .
$$

In the same way we have

$$
\left\|\left(\Delta_{L(G)}+\lambda_{+}\right) \phi f\right\|=\left.0 \Leftrightarrow f\right|_{V_{1}}=0 .
$$

And when $d_{1}=d_{2}=d, \lambda_{ \pm}=\frac{d}{D}$, and for any $f \in W_{1}$

$$
\left\|\left(\Delta_{L(G)}+\lambda_{ \pm}\right) \phi f\right\|=0 .
$$

This implies the second part of the theorem.

Next let us consider $-\left.\Delta_{L(G)}\right|_{E_{2}}$. Since $\phi^{*} F(x)=0$ for $F \in E_{2}$,

$$
\sum_{r \in N_{x}} F(x, r)=0 \quad \text { for any } x \in V(G),
$$


and so

$$
\begin{aligned}
- & \Delta_{L(G)} F(x, y) \\
& =\frac{-1}{d_{1}+d_{2}-2}\left(\sum_{r \in N_{x}}(F(x, r)-F(x, y))+\sum_{r \in N_{y}}(F(r, y)-F(x, y))\right) \\
& =\frac{d_{1}+d_{2}}{d_{1}+d_{2}-2} F(x, y) .
\end{aligned}
$$

Hence $\operatorname{Spec}\left(-\Delta_{L(G)} \mid E_{2}\right)=\left\{\frac{d_{1}+d_{2}}{d_{1}+d_{2}-2}\right\}$. Moreover, it can be shown that $E_{2}$ is infinite dimensional in the similar way as in Section 2.

\section{SUBDIVISION GRAPHS}

We define the subdivision of a graph $G$. The subdivision graph $S(G)$ of a graph $G$ is obtained from $G$ by replacing each edge by a path of length 2, or equivalently, by inserting an additional vertex into each edge of $G$ (see Figure 4.1).



G



$\mathrm{S}(\mathrm{G})$

FiguRE 4.1. Subdivision.

Remark 4.1. The subdivision graph of a $d$-regular graph is $(d, 2)$-semiregular.

Then we can show the following theorem:

Theorem 4.2. Let $d \geq 3$ and $G$ be a d-regular graph. Let $f_{ \pm}^{S}(x)=1 \pm \sqrt{1-x / 2}$. Then

$$
\operatorname{Spec}\left(-\Delta_{S(G)}\right)=f_{-}^{S}\left(\operatorname{Spec}\left(-\Delta_{G}\right)\right) \cup\{1\} \cup f_{+}^{S}\left(\operatorname{Spec}\left(-\Delta_{G}\right)\right)
$$

where 1 is an eigenvalue with infinite multiplicity.

We identify $V(S(G))$ with the set $V(G) \cup V(L(G))$. Then,

$$
E(S(G))=\left\{(x,(x, y)) \in V(G) \times V(L(G)) ; y \in N_{x} \text { in } G\right\} .
$$

The Laplacian $\Delta_{S(G)}$ is given by

$$
\begin{aligned}
\Delta_{S(G)} F(x, y) & =\frac{1}{2}(F(x)+F(y))-F(x, y), \\
\Delta_{S(G)} F(x) & =\frac{1}{d} \sum_{r \in N_{x}} F(x, r)-F(x) .
\end{aligned}
$$

We remark that $\ell^{2}(S(G))$ is identified with the direct sum $\ell^{2}(G) \oplus \ell^{2}(L(G))$ and so $F \in \ell^{2}(S(G))$ can be regarded as $F_{0}+F_{1} \in \ell^{2}(G) \oplus \ell^{2}(L(G))$. Two operators $\phi$ and $\phi^{*}$ defined in (2.1) are also useful in this section. 
Lemma 4.3. Let $\phi$ and $\phi^{*}$ be the same ones in (2.1). Let $F=F_{0}+F_{1} \in \ell^{2}(G) \oplus$ $\ell^{2}(L(G)) \cong \ell^{2}(S(G))$. Then $\Delta_{S(G)}$ has a matrix representation as follows:

$$
\Delta_{S(G)}\left(\begin{array}{c}
F_{0} \\
F_{1}
\end{array}\right)=\left(\begin{array}{cc}
-1 & \frac{C_{d}}{d} \phi^{*} \\
\frac{1}{2 C_{d}} \phi & -1
\end{array}\right)\left(\begin{array}{c}
F_{0} \\
F_{1}
\end{array}\right),
$$

where $C_{d}=\sqrt{\frac{d}{2 d-2}}$. Moreover,

$$
\left(\Delta_{S(G)}^{2}+2 \Delta_{S(G)}\right)\left(\begin{array}{c}
F_{0} \\
F_{1}
\end{array}\right)=\left(\begin{array}{cc}
\frac{1}{2} \Delta_{G} & 0 \\
0 & \frac{d-1}{d} \Delta_{L(G)}
\end{array}\right)\left(\begin{array}{c}
F_{0} \\
F_{1}
\end{array}\right) .
$$

Proof. We note that $\operatorname{supp} F_{0} \subset V(G)$ and $\operatorname{supp} F_{1} \subset V(L(G))$.

$$
\begin{aligned}
\Delta_{S(G)} F(x) & =\frac{1}{d} \sum_{r \in N_{x}} F(x, r)-F(x) \\
& =\frac{C_{d}}{d} \phi^{*} F_{1}(x)-F_{0}(x) .
\end{aligned}
$$

Similarly,

$$
\begin{aligned}
\Delta_{S(G)} F(x, y) & =\frac{1}{2}(F(x)+F(y))-F(x, y) \\
& =\frac{1}{2 C_{d}} \phi F_{0}(x, y)-F_{1}(x, y) .
\end{aligned}
$$

Then

$$
\Delta_{S(G)}\left(\begin{array}{c}
F_{0} \\
F_{1}
\end{array}\right)=\left(\begin{array}{cc}
-1 & \frac{C_{d}}{d} \phi^{*} \\
\frac{1}{2 C_{d}} \phi & -1
\end{array}\right)\left(\begin{array}{c}
F_{0} \\
F_{1}
\end{array}\right)
$$

and

$$
\Delta_{S(G)}^{2}=\left(\begin{array}{cc}
-1 & \frac{C_{d}}{d} \phi^{*} \\
\frac{1}{2 C_{d}} \phi & -1
\end{array}\right)^{2}=\left(\begin{array}{cc}
1+\frac{1}{2 d} \phi^{*} \phi & -\frac{2 C_{d}}{d} \phi^{*} \\
-\frac{1}{C_{d}} \phi & 1+\frac{1}{2 d} \phi \phi^{*}
\end{array}\right) .
$$

Hence using Lemma 2.4, we obtain

$$
\Delta_{S(G)}^{2}+2 \Delta_{S(G)}=\left(\begin{array}{cc}
\frac{1}{2} \Delta_{G} & 0 \\
0 & \frac{d-1}{d} \Delta_{L(G)}
\end{array}\right) .
$$

Proof of Theorem 4.2. Let $g(t)=-t^{2}+2 t$. By the spectral mapping theorem and Lemma 4.3, we have

$$
\begin{aligned}
g\left(\operatorname{Spec}\left(-\Delta_{S(G)}\right)\right) & =\operatorname{Spec}\left(g\left(-\Delta_{S(G)}\right)\right)=\operatorname{Spec}\left(-\left(\Delta_{S(G)}^{2}+2 \Delta_{S(G)}\right)\right) \\
& =\operatorname{Spec}\left(-\frac{1}{2} \Delta_{G}\right) \cup \operatorname{Spec}\left(-\frac{d-1}{d} \Delta_{L(G)}\right) \\
& =\operatorname{Spec}\left(-\frac{1}{2} \Delta_{G}\right) \cup\left(\operatorname{Spec}\left(-\frac{1}{2} \Delta_{G}\right) \cup\{1\}\right) .
\end{aligned}
$$

We used Theorem 2.2 in the third equality. Since $S(G)$ is bipartite, by the spectral mapping theorem and Lemma 3.5, we obtain

$$
\operatorname{Spec}\left(-\Delta_{S(G)}\right)=f_{-}^{S}\left(\operatorname{Spec}\left(-\Delta_{G}\right)\right) \cup\{1\} \cup f_{+}^{S}\left(\operatorname{Spec}\left(-\Delta_{G}\right)\right)
$$

where $f_{ \pm}^{S}$ are two branches of the inverse of $2 g$.

By Proposition 2.7, the eigenspace of $\{1\}$ (corresponding to $\left\{\frac{d}{d-1}\right\}$ of $-\Delta_{L(G)}$ ) is infinite dimensional. 


\section{PARA-LINE GRAPHS}

Let us define the para-line graph of a graph $G$. Given a graph $G$, insert two vertices to each edge $x y$ of $G$. Those two vertices will be denoted by $(x, y),(y, x)$, where $(x, y)$ (resp. $(y, x))$ is the one incident to $x$ (resp. $y$ ). We define the vertex set and the edge set as follows:

$$
\begin{aligned}
& V(p-L(G))=\{(x, y) \in V(G) \times V(G) ; x y \in E(G)\} . \\
& E(p-L(G))=\{((x, w),(x, z)) ;(x, w),(x, z) \in V(p-L(G)), w \neq z\} \\
& \cup\{((x, y),(y, x)) ; x y \in E(G)\} .
\end{aligned}
$$

The resultant graph is called a para-line graph and denoted by $p-L(G)$ (see Figure 5.1).

Remark 5.1. The para-line graph of a $d$-regular graph is $d$-regular and it can be regarded as the line graph of the subdivision of a graph.

Then we can show the following theorem.

Theorem 5.2. Let $d \geq 3$. Let $G$ be a d-regular graph and $p-L(G)$ its para-line graph, and $f_{ \pm}^{p}(x)=\left(d+2 \pm \sqrt{(d+2)^{2}-4 d x}\right) / 2 d$. Then

$$
\operatorname{Spec}\left(-\Delta_{p-L(G)}\right)=f_{-}^{p}\left(\operatorname{Spec}\left(-\Delta_{G}\right)\right) \cup\{1\} \cup f_{+}^{p}\left(\operatorname{Spec}\left(-\Delta_{G}\right)\right) \cup\left\{\frac{d+2}{d}\right\}
$$

where 1 and $\frac{d+2}{d}$ are eigenvalues with infinite multiplicity.

Now, by definition, $V(p-L(G))=\{(x, y) \in V(G) \times V(G) ; x y \in E(G)\}$. So, we can regard $\ell^{2}(p-L(G))$ as the space of square summable functions on the set above and write down $\Delta_{p-L(G)}$ as follows:

$$
\Delta_{p-L(G)} F(x, y)=\frac{1}{d}\left\{\sum_{r \in N_{x}}(F(x, r)-F(x, y))+(F(y, x)-F(x, y))\right\} .
$$

Proof of Theorem 5.2. We apply Theorem 3.2 to the subdivision of a $d$-regular graph $G$.

We identify $V(S(G))$ with $V(G) \cup V(L(G))$ as in the previous section. Put $V_{1}=V(G)$ and $V_{2}=V(L(G))$ in Theorem 3.2 and in this case $d_{1}=d, d_{2}=2$ and $D=d$.

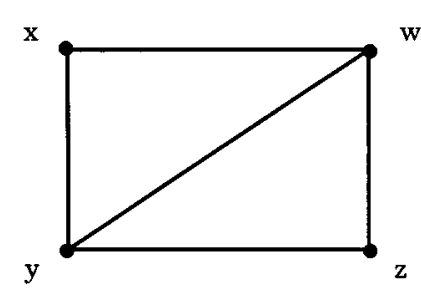

G

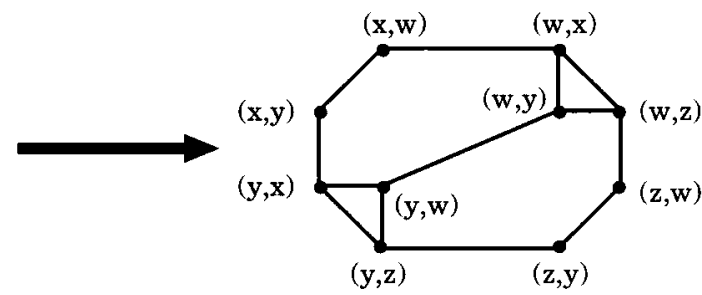

$\mathrm{p}-\mathrm{L}(\mathrm{G})$

Figure 5.1. Para-line graph. 
As mentioned in the introduction, by Remark 5.1 and Theorem 3.2, we have

$$
\begin{aligned}
& \operatorname{Spec}\left(-\Delta_{p-L(G)}\right) \\
& =f_{-}^{(d, 2)}\left(\overline{\operatorname{Spec}\left(-\Delta_{S(G)}\right) \backslash\{1\}}\right) \cup S \cup f_{+}^{(d, 2)}\left(\overline{\operatorname{Spec}\left(-\Delta_{S(G)}\right) \backslash\{1\}}\right) \cup\left\{\frac{d+2}{d}\right\} \\
& =f_{-}^{(d, 2)}\left(f_{ \pm}^{S}\left(\operatorname{Spec}\left(-\Delta_{G}\right)\right)\right) \cup S \cup f_{+}^{(d, 2)}\left(f_{ \pm}^{S}\left(\operatorname{Spec}\left(-\Delta_{G}\right)\right)\right) \cup\left\{\frac{d+2}{d}\right\} \\
& =f_{-}^{p}\left(\operatorname{Spec}\left(-\Delta_{G}\right)\right) \cup S \cup f_{+}^{p}\left(\operatorname{Spec}\left(-\Delta_{G}\right)\right) \cup\left\{\frac{d+2}{d}\right\},
\end{aligned}
$$

where $S \subset f_{ \pm}^{(d, 2)}(1)=\left\{\frac{d_{1}}{D}, \frac{d_{2}}{D}\right\}=\left\{1, \frac{2}{d}\right\}$. It is easy to check that $f_{ \pm}^{p}=f_{ \pm}^{(d, 2)} \circ f_{ \pm}^{S}$, where $f_{ \pm}^{(d, 2)}$ and $f_{ \pm}^{S}$ are the functions defined in Theorem 3.2 and Theorem 4.2. The set $S$ is not empty because the subdivision graph always has an eigenvalue $\{1\}$. So all we have to do is to obtain the information of the support of an eigenfunction for $\{1\}$ of $-\Delta_{S(G)}$.

Since 1 is not an eigenvalue of $-\frac{1}{2} \Delta_{G}$ by Remark 1.1, an eigenvalue $\{1\}$ comes only from $-\frac{d-1}{d} \Delta_{L(G)}$ in (4.2), and hence the support of each eigenfunction for $\{1\}$ is contained in $V_{2}$. Then, by Theorem $3.2, S=\left\{\frac{d_{1}}{D}\right\}=\{1\}$.

\section{Acknowledgement}

The author would like to thank Dr. Yu. Higuchi for his valuable suggestions and fruitful discussions. He also would like to thank Professor Y. Takahashi for his encouragement and suggestions.

\section{REFERENCES}

1. D. M. Cvetković, M. Doob and H. Sachs. Spectra of graphs - theory and application, Deutscher Verlag der Wissenschaften, Berlin, and Academic Press, New York, 1980. MR 81i:05054

2. J. Dodziuk and L. Karp. Spectral and function theory for combinatorial Laplacians, A. M. S. Contemporary Mathematics 73 (1988), 25-40. MR 89h:58220

3. Yu. Higuchi. Isoperimetric inequality and random walks on an infinite graph and its line graph, preprint.

4. Yu. Higuchi. Random Walks and Isoperimetric Inequalities on Infinite Planar Graphs and Their Duals, Dissertation, Univ. of Tokyo, January 1995.

5. B. Mohar and W. Woess. A survey of spectra of infinite graphs, Bull. London Math. Soc. 21 (1989), 209-234. MR 90d:05162

6. M. Reed and B. Simon. Methods of Modern Mathematical Physics, vol. I, Academic Press, New York, 1980. MR 85e:46002

7. T. Shima. On eigenvalue problems for the random walks on the Sierpinski pre-gasket, Japan J. Indust. Appl. Math., 8 (1991), 127-141. MR 92g:60094

Department of Mathematical Sciences, University of Tokyo, Komaba, Meguro-ku, TOKYO 153, JAPAN

Current address: Department of Mathematics, Tokyo Institute of Technology, Oh-okayama, Meguro-ku, Tokyo 152-8551, Japan

E-mail address: shirai@neptune.ap.titech.ac.jp 